M-N VAN THIENEN

Department of Medical Genetics, University of Antwerp, Belgium

K KEYMOLEN

Centre for Medical Genetics, University of Brussels, Belgium

1 Scambler PJ, Kelly D, Linsay E, et al. Velocardio-facial syndrome associated with chromosome 22 deletions encompassing the $\mathrm{Di}$ George locus. Lancet 1992;339:1138-9.

2 Driscoll DA, Salvin J, Sellinger B, et al. Prevalence of 22q11 microdeletions in DiPrevalence of 22q11 microdeletions in Diimplications for genetic counselling and prenatal diagnosis. $\mathcal{F}$ Med Genet 1993;30:813-17.

3 Wilson DI, Cross IE, Wren C, et al. Minimum prevalence of chromosome 22q11 deletions. Am ₹ Hum Genet 1994;55:A169.

4 Du Montcel ST, Mendizabal H, Aymé S, Lévy A, Philip N. Prevalence of 22q11 microdeletion. F Med Genet 1996;33:719.

\section{Sharing of PPT mutations between distinct clinical forms of neuronal ceroid lipofuscinoses in patients from Scotland}

The neuronal ceroid lipofuscinoses (NCLs, Batten disease) are a group of rare inherited neurodegenerative diseases of childhood classified according to their age of onset and ultrastructural appearance of storage material. A very unusual cluster of NCL cases is found in the west of Scotland. ${ }^{1}$ Some patients have early infantile onset, INCL, a disease almost entirely confined to Finland and characterised on ultrastructure by granular osmiophilic deposits (GROD). Other patients have a later, juvenile, onset but are found to have GROD (vJNCL/GROD) rather than the fingerprint profiles usually found in juvenile onset cases. INCL in Finland is caused by mutations in the gene encoding palmitoyl protein thioesterase, PPT. ${ }^{2}$ It was recently reported that cases of $\mathrm{vJNCL} / \mathrm{GROD}$ from Scotland (and elsewhere) also result from mutations in this gene. ${ }^{3}$ We set out to determine the disease causing mutations in PPT in Scottish INCL patients to establish whether there was any sharing of mutations with vJNCL/GROD.

Four patients with a diagnosis of INCL were analysed. Exons of PPT were amplified from genomic DNA by PCR and then sequenced in forward and reverse directions. ${ }^{3}$ We found mutations in PPT in all four patients and of the eight chromosomes analysed three different nonsense mutations (LeulOSTOP, Lys55STOP, and Arg151STOP, table 1) were present. Therefore, all the INCL patients are homozygous for mutations predicted to result in truncation of PPT. For each patient we amplified parental DNA and were able to show Mendelian inheritance of mutations.

Two of these mutations (Leu10STOP and Arg151STOP) are found in patients with vJNCL/GROD. ${ }^{3}$ However, in these cases nonsense mutations do not occur in homozygous form and are only found in combination with a missense mutation. Therefore, we can now show that two clinically distinct forms of NCL are caused by shared mutations in PPT. The clinical significance of these findings is that the severity of the disease in these patients is dependent on the combination and type of mutations present.

There are several diseases in which different mutations in the same gene cause dissimilar clinical phenotypes, for example, CFTR (cystic fibrosis and congenital bilateral absence of the vas deferens ${ }^{4}$ ). Types A and B Niemann-Pick disease, like the NCLs, are lysosomal storage disorders which are both caused by mutations in the acid sphingomyelinase gene. As in this study, the same mutation has been found in both forms and the age of onset and severity of the phenotype is dependent on the other allele. ${ }^{5}$

Geographical clustering of a rare autosomal recessive genetic disease suggests a founder effect with patients inheriting the same ancestral disease chromosomes. Detailed genealogical information is not available on these patients. However, all but one of the 18 disease chromosomes in INCL and vJNCL/GROD cases are accounted for by two nonsense and one missense mutations (table 1) and it is likely that these are derived from individual ancestral chromosomes. High resolution haplotype analysis and population studies to determine carrier rates will be required to resolve the issue.

We thank the Medical Research Council (PBM, NDEG) and Action Research (KYL) for financial sDEG)

PATRICIA B MUNROE NICHOLAS D E GREENE KIT-YI LEUNG SARA E MOLE R MARK GARDINER HANNAH M MITCHISON

Department of Paediatrics, University College London Medical School, Rayne Institute, 5 University Street, London WC1E 6FF, UK

JOHN B P STEPHENSON YANICK J CROW

Departments of Paediatric Neurology and Medical Genetics, Yorkhill Hospitals NHS Trust, Glasgow G3 8SF, UK

1 Crow YJ, Tolmie JL, Howatson AG, Patrick WJA, Stephenson JBP. Batten disease in the West of Scotland 1974-1995 including five cases of the juvenile form with granular osmiophilic deposits. Neuropaediatrics 1997;28:140-4.

2 Vesa J, Hellsten E, Verkruyse LA, et al. Mutations in the palmitoyl-protein thioesterase gene causing infantile neuronal ceroid lipofusgene causing infantile neuronal
cinosis. Nature 1995;376:584-7.

3 Mitchison HM, Hofmann SL, Becerra CHR, et al. Mutations in palmitoyl-protein thioesterase gene $(P P T ; C L N I)$ causing juvenile neuronal gene (PPT; CLNI) causing juvenile neuronal deposits. Hum Mol Genet 1998;7:291-7.

4 Chillon M, Casals T, Mercier B, et al. Mutations in the cystic-fibrosis gene in patients with conin the cystic-fibrosis gene in patients with congenital absence of the
Med 1995;332:1475-80.

Table 1 PPT mutations in Scottish INCL and vfNCL/GROD

\begin{tabular}{lll}
\hline Case & Disease & Mutations \\
\hline 389 & INCL & Arg151STOP/Arg151STOP \\
390 & INCL & Arg151STOP/Lys55STOP \\
392 & INCL & Arg151STOP/Leu10STOP \\
391 & INCL & Leu10STOP/Leu10STOP \\
$105,341,346$ & vJNCL/GROD & Arg151STOP/Thr75Pro \\
325,345 & vJNCL/GROD & Leu10STOP/Thr75Pro \\
\hline
\end{tabular}

Bold type indicates mutations present in both INCL and vJNCL/GROD.
5 Levran O, Desnick RJ, Schuchman EH Niemann-Pick type B disease. Identification of a single codon deletion in the acid sphingomyelinase gene and genotype/phenotype correlations in type A and B patients. $\mathcal{F}$ Clin Invest 1991;88:806-10.

\section{PTEN and prostate cancer}

The PTEN gene (phosphate and tensin homologue), located on $10 \mathrm{q} 23,{ }^{12}$ has been reported to be the Cowden disease susceptibility gene. Germline mutations in PTEN have been found in patients with this syndrome. ${ }^{3}$ This disorder is characterised by the development of hamartomas at various sites, as well as an increased predisposition for thyroid cancer and for breast cancer in women. ${ }^{4-6}$ PTEN has also been reported as being altered in other types of cancer including glioblastoma, ${ }^{12}$ endometrial carcinoma, ${ }^{7}$ and kidney carcinoma. ${ }^{2}$ This gene has additionally been suggested to play a role in prostate cancer as PTEN alterations have been found in multiple prostate cancer cell lines. ${ }^{1}$

In order to investigate the role of mutation in the PTEN gene in primary prostate cancer, we analysed microdissected prostate adenocarcinoma tissue from 28 patients with histopathologically confirmed cancer. All nine exons of PTEN were PCR amplified and screened for mutations by single strand conformational polymorphism analysis (SSCP). Samples displaying mobility shifts were subjected to DNA sequence analysis. This analysis failed to detect homozygous deletions of the PTEN gene in any sample. A heterozygous mutation was identified in only one of the prostate tumour samples and was charac terised as a single base deletion in codon 68 (TAC $\rightarrow \mathrm{AC}$ ). Additionally, an $\mathrm{A} \rightarrow \mathrm{G}$ polymorphism 96 bp upstream of the beginning of exon 2 was found in nine of 28 samples $(32.1 \%)$. Based on this analysis, we conclude that mutations of the PTEN gene are rare in primary prostate cancers.

EVAN A FACHER Department of Epidemiology and Biostatistics, Case Western Reserve University, 2500 MetroHealth Drive Rammelkamp-R459, Cleveland, OH 44109, USA JOHN C LAW

Department of Human Genetics, University of Pittsburgh, USA

$1 \mathrm{Li} \mathrm{J}$, Yen C, Liaw D, et al. PTEN, a putative protein tyrosine phosphatase gene mutated in human brain, breast, and prostate cancer. Science 1997;275:1943-7.

2 Steck PA, Pershouse MA, Jasser SA, et al. Identification of a candidate tumour suppressor gene, MMAC1, at chromosome $10 \mathrm{q} 23.3$ that is mutated in multiple advanced cancers. Nat Genet 1997; 15:356-62.

3 Liaw D, Marsh DJ, Li J, et al. Germline mutations of the PTEN gene in Cowden mutations of the PTEN gene in Cowden syndrome. Nat Genet 1997;16:64-7.

4 Carlson HE, Burns TW, Davenport SL, et al Cowden disease: gene marker studies and measurements of epidermal growth factor. $A m$ f Hum Genet 1986;38:908-17.

5 Starink TM, van der Veen JP, Arwert F, et al. The Cowden syndrome: a clinical and genetic study in 21 patients. Clin Genet 1986;29:222 33.

6 Eng C, Murday V, Seal S, et al. Cowden syndrome and Lhermitte-Duclos disease in family: a single genetic syndrome with pleiotropy? f Med Genet 1994;31:458-61.

7 Kong D, Suzuki A, Zou TT, et al. PTEN1 is frequently mutated in primary endometrial carciquently mutated in primary endom

8 Tashiro H, Blazes MS, Wu R, et al. Mutations in PTEN are frequent in endometrial carcinoma but rare in other common gynecological malignancies. Cancer Res 1997;57:3935-40. 